\title{
The research training and education programs in the optical and photonic fields at Fudan University
}

\section{Liang-Yao Chen}

Liang-Yao Chen, "The research training and education programs in the optical and photonic fields at Fudan University," Proc. SPIE 9663, Eighth International Topical Meeting on Education and Training in Optics and Photonics, 96631D (6 October 2003); doi: 10.1117/12.2208463

SPIE Event: Eighth International Topical Meeting on Education and Training in Optics and Photonics, 2003, Tucson, Arizona, United States 


\title{
The research training and education programs in the optical and photonic fields at Fudan University
}

\author{
Liang-Yao Chen \\ The State Key Lab for Advanced Photonics Materials and Devices, Department of Optical Science and Engineering, Fudan University, \\ Shanghai, 200433, China \\ telephone/fax:086-21-6564-6068; e-mail:lychen@fudan.ac.cn
}

\begin{abstract}
Facing great challenge in the optical and photonic fileds, the department of optical science and engineering at Fudan university has carried out unique research training and education programs with multiple levels to have students obtain broad knowledge and research training experience by attending the class studies and active research projects.

(C)2003 Optical Society of America

OCIS codes: (000.2060) Education
\end{abstract}

\begin{abstract}
Summary
In the 21 century, the information science and technology will continue to achieve great progress with wide applications in many fields, which will have significant influence on our society. This is particularly true for the fast and advanced development in the optical and photonics fields with important application, for example, in the optical communication. Instead of using electrons, the photons with multiple wavelengths will carry and process the rich information more effectively in the modern broadband network system. Accompanied with the fast developing steps, however, we are facing great challenge in the research training and education program in the optical and photonic fileds that show increasing demand of highly specialized and qualified research scientists and engineers to work in the academic institutes and industry in the future, with the ability to solve both present and future problems in the fields. With strong support from the university, those professors having physics background and research experience in the optical and photonic fields come to establish a new department of optical science and engineering. It is also in the frame of the state key laboratory of advanced photonics materials and devices. There are multiple levels of research training and education programs in the department. For those students to complete the undergraduate training program, they will obtain broad knowledge by taking the classes in mathematics, fundamental physics, quantum mechanics, modern optics and so on. After graduated with a BS degree, some of them will continue to be trained in the research level through the MS and Ph. D. programs. They will take more advanced classes related to the optics and photonics. They will not only study the fundamental principle of optics with material and device development and applications, but also at the same time will very actively take part in research projects through intensive training processes. They will read extensive research papers, try to establish the experiment system most independently by themselves, acquire and analyze the data correctly, have the practice to give presentation in the seminar and conference regularly, and write papers with sound results to publish in the research journals finally. The research activities carried out in the department have a wide range from theoretic to experimental studies in optics and photonics, such as nonlinear optics, high power laser device and application, ultra-fast (fs) optical process, optical and magneto-optical properties of materials, photonic crystals, thin films, nano-optics, optical materials and devices used in broadband communication, and so on. The research and education in the optical and photonic fields at Fudan also have cross-disciplines with other fields of physics, micro-electronics, material science to have students get broader knowledge and research training experience. The program will help students to have the solid confidence in mind for their future career lives to work in this prospering field in the coming new age.
\end{abstract}

Eighth International Topical Meeting on Education and Training in Optics and Photonics, edited by Barry L. Shoop, Grover Swartzlander Jr., Proc. of SPIE Vol. 9663, 96631D

(c) 2003 SPIE, OSA, ICO · doi: 10.1117/12.2208463 